\title{
Dynamics Analysis of an HIV Infection Model including Infected Cells in an Eclipse Stage
}

\author{
Shengyu Zhou, Zhixing Hu, Wanbiao Ma, and Fucheng Liao \\ Department of Applied Mathematics, University of Science and Technology Beijing, Beijing 100083, China \\ Correspondence should be addressed to Zhixing Hu; huzhixing@ustb.edu.cn
}

Received 28 January 2013; Accepted 31 March 2013

Academic Editor: Junjie Wei

Copyright ( 2013 Shengyu Zhou et al. This is an open access article distributed under the Creative Commons Attribution License, which permits unrestricted use, distribution, and reproduction in any medium, provided the original work is properly cited.

\begin{abstract}
In this paper, an HIV infection model including an eclipse stage of infected cells is considered. Some quicker cells in this stage become productively infected cells, a portion of these cells are reverted to the uninfected class, and others will be latent down in the body. We consider CTL-response delay in this model and analyze the effect of time delay on stability of equilibrium. It is shown that the uninfected equilibrium and CTL-absent infection equilibrium are globally asymptotically stable for both ODE and DDE model. And we get the global stability of the CTL-present equilibrium for ODE model. For DDE model, we have proved that the CTL-present equilibrium is locally asymptotically stable in a range of delays and also have studied the existence of Hopf bifurcations at the CTL-present equilibrium. Numerical simulations are carried out to support our main results.
\end{abstract}

\section{Introduction}

In recent years, mathematical models have been done on the viral dynamics of HIV. In the basic mathematical modeling of viral dynamics, the description of the virus infection process has three populations: uninfected target cells, productively infected cells, and free viral particles [1-7]. In this model, infected cells are assumed to produce new virions immediately after target cells are infected by a free virus.

However, there are many biological steps between viral infection of target cells and the production of HIV-1 virions. In 2007, Rong and coworkers [8] studied an extension of the basic model of HIV-1 infection. The main feature of their model is that an eclipse stage for the infected cells is included and a portion of these cells are reverted to the uninfected class. Perelson et al. [9] presented this kind of cell early in 1993. Buonomo and Vergas-De-León [10] have performed the global stability analysis of this model. Perelson et al. [1] put forward another model in 1997. He divided infected cells into two kinds: long-lived productively infected cells and latently infected cells. Latently infected cells are also activated into productively infected cells [11]. Motivated by their work and now we concern the progression of infected cells from this eclipse phase to the productive, and a portion of these cells are reverted to the uninfected class or are latent down in the body.
In most virus infections, cytotoxic T lymphocytes (CTLs) play a critical role in antiviral defense by attacking virusinfected cells. Therefore, the dynamics of HIV infection with CTL response has received much attention in the past decades, some include the immune response without immune delay [12-15], and others contain immune delay [16-19]. Some HIV infection models with CTL-response describe only the interaction among uninfected target cells, productively infected cells, CTLs $[12,14,20]$. The most basic model can be written as

$$
\begin{aligned}
& \frac{d x}{d t}=s-d x-\beta x y, \\
& \frac{d y}{d t}=\beta x y-\alpha y-p y z, \\
& \frac{d z}{d t}=f(x, y, z)-r z,
\end{aligned}
$$

where $x, y$, and $z$ represent the concentration of uninfected target cells, productively infected cells, CTLs at time $t$, respectively. Parameters $s$ and $d$ are the birth rate and death rate of uninfected cells, respectively. The uninfected cells become infected at rate of $\beta x y$. Productively infected cells are produced at rate $\beta x y, \alpha$ is the death rate of productively 
infected cells, $p$ is the strength of the lytic component, and $r$ is the death rate of CTLs. Function $f(x, y, z)$ describes the rate of immune response activated by the infected cells. Wang et al. [14] assumed that the production of CTLs depends only on the population of infected cells and gave $f(x, y, z)=c y$. Ji et al. [12] assumed that the production of CTLs also depends on the population of CTL cells and chose the former $f(x, y, z)=$ cyz.

In this paper, we also consider the dynamics of HIV infection with CTL response and give $f(x, y, z)=c y z$. Meanwhile, our model also concludes an eclipse stage of infected cells. After the eclipse stage, some quicker infected cells which become productively infected cells are obviously attacked by CTLs. Other infected cells which will be reverted to the uninfected class or be latent down in the body do not have the ability to express HIV and will not cause CTL immune response. Therefore, we only take the immune response to productively infected cells into account and ignore the attack to latently infected cells by CTLs. So we get the following ODE:

$$
\begin{gathered}
\frac{d x}{d t}=s-\beta x y-d x+\delta w, \\
\frac{d w}{d t}=\beta x y-(\delta+\eta+q) w, \\
\frac{d y}{d t}=q w-p y z-\alpha y, \\
\frac{d z}{d t}=c y z-r z,
\end{gathered}
$$

where $w$ represents the concentration of infected cells in the eclipse stage at time $t$. Infected cells in the eclipse phase revert to the uninfected class at a constant rate $\delta$. In addition, they may alternatively progress to the productively infected class at the rate $q$ or die at the rate $\eta$. But some authors believe that time delay cannot be ignored in models for immune response [16-19]. In this paper, $\tau$ represents CTL-response delay, that is, the time between antigenic stimulation and generating CTLs. We investigated the effect of a time delay on system (2) to obtain the following DDE model:

$$
\begin{gathered}
\frac{d x}{d t}=s-\beta x y-d x+\delta w, \\
\frac{d w}{d t}=\beta x y-(\delta+\eta+q) w, \\
\frac{d y}{d t}=q w-p y z-\alpha y, \\
\frac{d z}{d t}=c y(t-\tau) z(t-\tau)-r z .
\end{gathered}
$$

Our paper is organized as follows: the three equilibriums on system (2) and (3) are given in the next section. In Section 3, the global stability of the ODE model is discussed. The analysis of the stability for this DDE model is carried out in Section 4. Finally, some numerical simulations are carried out to support our analytical results, and some conclusions are presented.

\section{The Existence of the Equilibrium of System}

In system (2) and (3), the basic reproduction numbers for viral infection and for CTL response are given as follows:

$$
\begin{aligned}
& R_{0}=\frac{q s \beta}{d \alpha(\delta+\eta+q)}, \\
& R_{1}=\frac{c q s \beta}{c d \alpha(\delta+\eta+q)+\alpha \beta r(\eta+q)} .
\end{aligned}
$$

It is clear that $R_{0}>R_{1}$ always holds. For system (2) and (3), there exists three equilibriums.

Theorem 1. For system (2) and (3), the uninfected equilibrium $E_{0}(s / d, 0,0,0)$ always exists;

(1) if $R_{0}>1$, a CTL-absent infection equilibrium $E_{1}\left(x_{1}, w_{1}, y_{1}, z_{1}\right)$ exists, where

$$
\begin{aligned}
& x_{1}=\frac{\alpha(\delta+\eta+q)}{\beta q}, \\
& w_{1}=\frac{s}{n+q}\left(1-\frac{1}{R_{0}}\right), \\
& y_{1}=\frac{q}{\alpha} w_{1}, \quad z_{1}=0 ;
\end{aligned}
$$

(2) if $R_{1}>1$, there exists a CTL-present infection equilibrium $E_{2}\left(x_{2}, w_{2}, y_{2}, z_{2}\right)$, where

$$
\begin{aligned}
& x_{2}=\frac{c(\delta+\eta+q)}{\beta r} w_{2}, \\
& w_{2}=\frac{s \beta r}{c d(\delta+\eta+q)+\beta r(\eta+q)}, \\
& y_{2}=\frac{r}{c}, \quad z_{2}=\frac{\alpha}{p}\left(R_{1}-1\right) .
\end{aligned}
$$

\section{The Global Stability of the ODE Model}

The initial conditions for system (2) are given as follows:

$$
x(0)>0, \quad w(0)>0, \quad y(0)>0, \quad z(0)>0 .
$$

It is clear that all solutions of system (2) are positive for $t>0$. Before analyzing the stability of system (2), we now show that the solutions of system (2) are bounded.

Theorem 2. Let $(x(t), w(t), y(t)$, and $z(t))$ be the solution of system (2) satisfying initial conditions (7), then there exists $M>0$ such that $x(t)<M, w(t)<M, y(t)<M$, and $z(t)<M$ hold after sufficiently large time $t$.

Proof. Let

$$
L(t)=x(t)+w(t)+y(t)+\frac{p}{c} z(t),
$$


where $k=\min \{d, \eta, \alpha, r\}$. It follows from (2) that

$$
\begin{aligned}
\frac{d L(t)}{d t} & =s-d x(t)-\eta w(t)-\alpha y(t)-\frac{p r}{c} z(t) \\
& <s-k\left(x(t)+w(t)+y(t)+\frac{p}{c} z(t)\right) \\
& =s-k L(t) .
\end{aligned}
$$

Therefore, $L(t)<s / k+\varepsilon$ for all large $t$, where $\varepsilon$ is an arbitrarily small positive constant. Thus, $x(t)<M, w(t)<M, y(t)<M$ and $z(t)<M$ for some positive constant $M$.

Theorem 3. If $R_{0}<1$, the uninfected equilibrium $E_{0}$ of system (2) is globally asymptotically stable.

Proof. Construct a Lyapunov function

$$
\begin{aligned}
V_{1}(x, w, y, z)= & x-x_{0}-x_{0} \ln \frac{x}{x_{0}} \\
& +\frac{\delta}{2(d+\eta+q) x_{0}}\left[\left(x-x_{0}\right)+w\right]^{2} \\
& +w+\frac{(\delta+\eta+q)}{q} y+\frac{p(\delta+\eta+q)}{c q} z,
\end{aligned}
$$

where $x_{0}=s / d$. The derivative of $V_{1}$ along positive solutions of system (2) is given as follows:

$$
\begin{aligned}
\frac{d V_{1}}{d t}= & \frac{\left(x-x_{0}\right)}{x}(s-d x-\beta x y+\delta w) \\
& +\frac{\delta}{(d+\eta+q) x_{0}}\left(x-x_{0}+w\right) \\
& \times[s-d x-(\eta+q) w] \\
& +\frac{(\delta+\eta+q)}{q}(q w-\alpha y-p y z) \\
& +\beta x y-(\delta+\eta+q) w \\
& +\frac{p(\delta+\eta+q)}{q} y z-\frac{\operatorname{pr}(\delta+\eta+q)}{c q} z .
\end{aligned}
$$

On substituting $s=d x_{0}$ and $\delta w\left(x-x_{0}\right) / x=$ $-\delta w\left(\left(x-x_{0}\right)^{2} / x x_{0}\right)+\delta w\left(\left(x-x_{0}\right) / x_{0}\right)$ into (11), we derive that

$$
\begin{aligned}
\frac{d V_{1}}{d t}= & -\left(d x_{0}+\delta w+\frac{d \delta x}{d+\eta+q}\right) \frac{\left(x-x_{0}\right)^{2}}{x x_{0}} \\
& -\frac{\delta(\eta+q)}{(d+\eta+q) x_{0}} w^{2} \\
& +\frac{\alpha(\delta+\eta+q)}{q}\left(R_{0}-1\right) y \\
& -\frac{p r(\delta+\eta+q)}{c q} z .
\end{aligned}
$$

If $R_{0}<1$, then $d V_{1} / d t \leq 0$ for all $x>0, w>0, y>0$ and $z>$ 0 . So the uninfected equilibrium $E_{0}$ is stable. Clearly, it follows from (12) that $d V_{1} / d t=0$ if and only if $x=x_{0}=s / d, w=$ $0, y=0$, and $z=0$. Therefore, the largest invariant set in the set $\left\{(x, w, y, z) \in R_{+}^{4} \mid d V_{1} / d t=0\right\}$ is the singleton $\left\{E_{0}\right\}$. By LaSalle invariance principle, it follows that the equilibrium $E_{0}$ is globally asymptotically stable.

Theorem 4. For system (2), if $R_{1}<1$ and $1<R_{0} \leq$ $1+(\eta+q) / \delta$, CTL-absent infection equilibrium $E_{1}$ is globally asymptotically stable.

Proof. Define a Lyapunov function

$$
\begin{aligned}
V_{2}(x, w, y, z)= & x-x_{1}-x_{1} \ln \frac{x}{x_{1}} \\
& +w-w_{1}-w_{1} \ln \frac{w}{w_{1}}+\frac{\beta p x_{1} y_{1}}{c q w_{1}} z \\
& +\frac{\delta}{2(d+\eta+q) x_{1}}\left[\left(x-x_{1}\right)+\left(w-w_{1}\right)\right]^{2} \\
& +\frac{\beta x_{1} y_{1}}{q w_{1}}\left(y-y_{1}-y_{1} \ln \frac{y}{y_{1}}\right) .
\end{aligned}
$$

Calculating the derivative of $V_{2}$ along positive solutions of system (2), it follows that

$$
\begin{aligned}
\frac{d V_{2}}{d t}= & \left(1-\frac{x_{1}}{x}\right) \frac{d x}{d t}+\frac{\beta x_{1} y_{1}}{q w_{1}}\left(1-\frac{y_{1}}{y}\right) \frac{d y}{d t} \\
& +\frac{\delta}{(d+\eta+q) x_{1}}\left[\left(x-x_{1}\right)+\left(w-w_{1}\right)\right] \\
& \times\left(\frac{d x}{d t}+\frac{d w}{d t}\right)+\left(1-\frac{w_{1}}{w}\right) \frac{d w}{d t}+\frac{\beta p x_{1} y_{1}}{q c w_{1}} \frac{d z}{d t} \\
= & \left(1-\frac{x_{1}}{x}\right)(s-d x-\beta x y+\delta w) \\
& +\frac{\beta x_{1} y_{1}}{q w_{1}}\left(1-\frac{y_{1}}{y}\right)(q w-\alpha y-p y z) \\
& +\frac{\delta}{(d+\eta+q) x_{1}}\left[\left(x-x_{1}\right)+\left(w-w_{1}\right)\right] \\
& +\frac{\beta p x_{1} y_{1}}{q w_{1}} y z-\frac{\beta p r x_{1} y_{1}}{c q w_{1}} z . \\
& +\left(1-\frac{w_{1}}{w}\right)[\beta x y-(\delta+\eta+q) w] \\
& {[s-(\eta+q) w] } \\
& (1 x-(\delta) \\
&
\end{aligned}
$$


At CTL-absent infection equilibrium $E_{1}$, on substituting $s=$ $\beta x_{1} y_{1}+d x_{1}-\delta w_{1}, \alpha=q\left(w_{1} / y_{1}\right)$, and $\delta+\eta+q=\beta x_{1} y_{1} / w_{1}$ into (14), we obtain that

$$
\begin{aligned}
\frac{d V_{2}}{d t} & \\
= & \left(1-\frac{x_{1}}{x}\right)\left[-d\left(x-x_{1}\right)-\beta x y+\beta x_{1} y_{1}+\delta\left(w-w_{1}\right)\right] \\
& +\frac{\beta x_{1} y_{1}}{q w_{1}}\left(1-\frac{y_{1}}{y}\right)\left(q w-q w_{1} \frac{y}{y_{1}}-p y z\right) \\
& +\frac{\delta}{(d+\eta+q)}\left[\left(x-x_{1}\right)+\left(w-w_{1}\right)\right] \\
& \times\left[-d\left(x-x_{1}\right)-(\eta+q)\left(w-w_{1}\right)\right] \\
& +\left(1-\frac{w_{1}}{w}\right)\left(\beta x y-\beta x_{1} y_{1} \frac{w}{w_{1}}\right) \\
& +\frac{\beta p x_{1} y_{1}}{q w_{1}} y z-\frac{\beta r p x_{1} y_{1}}{c q w_{1}} z .
\end{aligned}
$$

Noting that

$$
\begin{aligned}
& \delta\left(1-\frac{x}{x_{1}}\right)\left(w-w_{1}\right) \\
& =-\delta\left(w-w_{1}\right) \frac{\left(x-x_{1}\right)^{2}}{x x_{1}}+\frac{\delta}{x_{1}}\left(x-x_{1}\right)\left(w-w_{1}\right),
\end{aligned}
$$

therefore,

$$
\begin{aligned}
\frac{d V_{2}}{d t} & \\
= & -\left(d x_{1}-\delta w_{1}+\delta w+\frac{d \delta x}{d+\eta+q}\right) \frac{\left(x-x_{1}\right)^{2}}{x x_{1}} \\
& -\frac{\delta(\eta+q)}{(d+\eta+q)}\left(w-w_{1}\right)^{2} \\
& -\beta x_{1} y_{1}\left(\frac{x_{1}}{x}+\frac{y_{1} w}{y w_{1}}+\frac{x y w_{1}}{x_{1} y_{1} w}-3\right) \\
& +\frac{\beta p x_{1} y_{1}}{c q w_{1}}\left[\frac{d(\delta+\eta+q)+r \beta(\eta+q)}{\beta(\eta+q)}\right]\left(R_{1}-1\right) z .
\end{aligned}
$$

Since $x_{1} / x+y_{1} w / y w_{1}+x y w_{1} / x_{1} y_{1} w-3 \geq 0$ and the equality holds if and only if $x=x_{1}, w=w_{1}$ and $y=y_{1}$. If $R_{0} \leq$ $1+(\eta+q) / \delta$, then $d x_{1}-\delta w_{1} \geq 0$. So, if $R_{1}<1$ and $1<R_{0} \leq$ $1+(\eta+q) / \delta$, then $d V_{2} / d t \leq 0$ for all $x>0, w>0, y>0$ and $z>0$. Clearly, it follows from (17) that $d V_{2} / d t=0$ if and only if $x=x_{1}, w=w_{1}, y=y_{1}$, and $z=0$, thus the largest invariant set in the set $\left\{(x, w, y, z) \in R_{+}^{4} \mid d V_{2} / d t=0\right\}$ is the singleton $\left\{E_{1}\right\}$. Therefore, the global asymptotic stability of $E_{1}$ follows from the LaSalle's invariance principle.
Theorem 5. For system (2), if $R_{1}>1$ and $c d(\delta+\eta+q)-$ $r \beta \delta \geq 0$, CTL-present infection equilibrium $E_{2}$ is globally asymptotically stable.

Proof. Define a Lyapunov function

$$
\begin{aligned}
& V_{3}(x, w, y, z) \\
& =x-x_{2}-x_{2} \ln \frac{x}{x_{2}}+w-w_{2}-w_{2} \ln \frac{w}{w_{2}} \\
& \quad+\frac{\delta}{2(d+\eta+q) x_{2}}\left[\left(x-x_{2}\right)+\left(w-w_{2}\right)\right]^{2} \\
& +\frac{\beta x_{2} y_{2}}{q w_{2}}\left(y-y_{2}-y_{2} \ln \frac{y}{y_{2}}\right) \\
& +\frac{\beta p x_{2} y_{2}}{c q w_{2}}\left(z-z_{2}-z_{2} \ln \frac{z}{z_{2}}\right) .
\end{aligned}
$$

Calculating the derivative of $V_{3}$ along positive solutions of system (2), we obtain that

$$
\begin{aligned}
\frac{d V_{3}}{d t} & \left(1-\frac{x_{2}}{x}\right) \frac{d x}{d t}+\frac{\beta x_{2} y_{2}}{q w_{2}}\left(1-\frac{y_{2}}{y}\right) \frac{d y}{d t} \\
& +\frac{\delta}{(d+\eta+q) x_{2}}\left[\left(x-x_{2}\right)+\left(w-w_{2}\right)\right]\left(\frac{d x}{d t}+\frac{d w}{d t}\right) \\
& +\left(1-\frac{w_{2}}{w}\right) \frac{d w}{d t}+\frac{\beta p x_{2} y_{2}}{q c w_{2}}\left(1-\frac{z_{2}}{z}\right) \frac{d z}{d t} \\
= & \left(1-\frac{x_{2}}{x}\right)(s-d x-\beta x y+\delta w) \\
& +\frac{\beta x_{2} y_{2}}{q w_{2}}\left(1-\frac{y_{2}}{y}\right)(q w-\alpha y-p y z) \\
& +\frac{\delta}{(u+\eta+q) x_{2}}\left[\left(x-x_{2}\right)+\left(w-w_{2}\right)\right] \\
& \times[s-d x-(\eta+q) w] \\
& +\left(1-\frac{w_{2}}{w}\right)[\beta x y-(\delta+\eta+q) w] \\
q c w_{2} & \left(1-\frac{z_{2}}{z}\right)(c y z-r z) .
\end{aligned}
$$

At CTL- present infection equilibrium $E_{2}$, on substituting $s=$ $\beta x_{2} y_{2}+d x_{2}-\delta w_{2}, \alpha=q\left(w_{2} / y_{2}\right)-p z_{2}, y_{2}=r / c$, and $\delta+\eta+q=$ $\beta x_{2} y_{2} / w_{2}$ into (19), it follows that

$$
\begin{aligned}
\frac{d V_{3}}{d t}= & \left(1-\frac{x_{2}}{x}\right) \\
& \times\left[-d\left(x-x_{2}\right)-\beta x y+\beta x_{2} y_{2}+\delta\left(w-w_{2}\right)\right]
\end{aligned}
$$




$$
\begin{aligned}
& +\frac{\beta x_{2} y_{2}}{q w_{1}}\left(1-\frac{y_{2}}{y}\right)\left(q w-q w_{2} \frac{y}{y_{2}}+p y z_{2}-p y z\right) \\
& +\frac{\delta}{(d+\eta+q)}\left[\left(x-x_{2}\right)+\left(w-w_{2}\right)\right] \\
& \times\left[-d\left(x-x_{2}\right)-(\eta+q)\left(w-w_{2}\right)\right] \\
& +\left(1-\frac{w_{2}}{w}\right)\left(\beta x y-\beta x_{2} y_{2} \frac{w}{w_{2}}\right) \\
& +\frac{\beta p x_{2} y_{2}}{q c w_{2}}\left(1-\frac{z_{2}}{z}\right)(c y z-r z) .
\end{aligned}
$$

Noting that

$$
\begin{aligned}
\delta\left(1-\frac{x}{x_{2}}\right)\left(w-w_{2}\right)= & -\delta\left(w-w_{2}\right) \frac{\left(x-x_{2}\right)^{2}}{x x_{2}} \\
& +\frac{\delta}{x_{2}}\left(x-x_{2}\right)\left(w-w_{2}\right),
\end{aligned}
$$

it follows from (20) and (21) that

$$
\begin{aligned}
\frac{d V_{3}}{d t}= & -\left(d x_{2}+\delta\left(w-w_{2}\right)\right) \frac{\left(x-x_{2}\right)^{2}}{x x_{2}} \\
& +\frac{\delta}{x_{2}}\left(x-x_{2}\right)\left(w-w_{2}\right) \\
& +\beta x_{2} y_{2}\left(1-\frac{x y}{x_{2} y_{2}}-\frac{x_{2}}{x}+\frac{y}{y_{2}}\right) \\
& +\beta x_{2} y_{2}\left(\frac{w}{w_{2}}-\frac{y}{y_{2}}-\frac{y_{2} w}{y w_{2}}+1\right) \\
& -\frac{\beta p x_{2} y_{2}}{q w_{2}}\left(y-y_{2}\right)\left(z-z_{2}\right) \\
& -\frac{\delta}{x_{2}}\left[\frac{d}{d+\eta+q}\left(x-x_{2}\right)^{2}+\left(x-x_{2}\right)\right. \\
& +\beta x_{2} y_{2}\left(\frac{x y}{x_{2} y_{2}}-\frac{w}{w_{2}}-\frac{x w_{2} y}{x_{2} w y_{2}}+1\right) \\
& +\frac{\beta p x_{2} y_{2}}{q w_{2}}\left(z-z_{2}\right)\left(y-y_{2}\right) \\
= & -\left(d x_{2}-\delta w_{2}+\delta w+\frac{\eta+q}{d+\eta+q}\right) \frac{\left(x-x_{2}\right)^{2}}{x x_{2}} \\
& -\frac{\delta(\eta+q)}{(d+\eta+q)}\left(w-w_{2}\right)^{2} \\
& -\beta x_{2} y_{2}\left(\frac{x_{2}}{x}+\frac{y_{2} w}{y w_{2}}+\frac{x w_{2} y}{x_{2} y_{2} w}-3\right) . \\
& \times(w-\eta) \\
&
\end{aligned}
$$

Since $x_{2} / x+y_{2} w / y w_{2}+x w_{2} y / x_{2} y_{2} w-3 \geq 0$ and the equality holds if and only if $x=x_{2}, w=w_{2}$, and $y=y_{2}$. If $c d(\delta+\eta+$ $q)-r \beta \delta \geq 0$, then $d x_{2}-\delta w_{2}=((c d(\delta+\eta+q)-r \beta \delta) / r \beta) w_{2} \geq 0$. Therefore, if $R_{1}>1$ and $c d(\delta+\eta+q)-r \beta \delta \geq 0$, it follows from (22) that $d V_{3} / d t \leq 0$ for all $x>0, w>0, y>0$, and $z>0$. Clearly, it follows from (22) that $d V_{3} / d t=0$ if and only if $x=x_{2}, w=w_{2}, y=y_{2}$, and $z=z_{2}$. So the largest invariant set in the set $\left\{(x, w, y, z) \in R_{+}^{4} \mid d V_{3} / d t=0\right\}$ is the singleton $\left\{E_{2}\right\}$. By LaSalle invariance principle, we conclude that the equilibrium $E_{2}$ is globally asymptotically stable.

\section{The Stability Analysis of the DDE Model}

In this section, we consider the stability of the delay model (3).

Let $C=C\left([-\tau, 0], R_{+0}^{4}\right)$ be the Banach space of continuous functions mapping from the interval $[-\tau, 0]$ to $R_{+0}^{4}$ with the topology of uniform convergence, where

$$
R_{+0}^{4}=\left\{\left(x_{1}, x_{2}, x_{3}, x_{4}\right) \mid x_{i} \geq 0, i=1,2,3,4\right\} .
$$

The initial conditions for system (3) are given as follows:

$$
\begin{gathered}
x=\varphi_{1}(\theta), \quad w=\varphi_{2}(\theta), \quad y=\varphi_{3}(\theta), \\
z=\varphi_{4}(\theta), \quad \theta \in[-\tau, 0], \\
x(0)>0, \quad w(0)>0, \quad y(0)>0, \quad z(0)>0,
\end{gathered}
$$

where $\left(\varphi_{1}(\theta), \varphi_{2}(\theta), \varphi_{3}(\theta), \varphi_{4}(\theta)\right) \in C\left([-\tau, 0], R_{+0}^{4}\right)$. It is clear to see that all solutions of system (3) satisfying the initial conditions (24) are positive for all $t \geq 0$. By the similar method to Theorem 2, we can get the following theorem.

Theorem 6. Let $x(t), w(t), y(t)$, and $z(t)$ be the solution of system (3) satisfying the initial conditions (24), then there exists $M>0$ such that $x(t)<M, w(t)<M, y(t)<M$, and $z(t)<$ $M$ hold after sufficiently large time $t$.

Theorem 7. If $R_{0}<1$, the uninfected equilibrium $E_{0}$ of system (3) is globally asymptotically stable.

Proof. If $R_{0}<1$, construct a Lyapunov functional

$$
\begin{aligned}
W_{1}(x, w, y, z)= & x-x_{0}-x_{0} \ln \frac{x}{x_{0}} \\
& +\frac{\delta}{2(d+\eta+q) x_{0}}\left[\left(x-x_{0}\right)+w\right]^{2}+w \\
& +\frac{(\delta+\eta+q)}{q} y+\frac{p(\delta+\eta+q)}{c q} z \\
& +\frac{p(\delta+\eta+q)}{q} \int_{t-\tau}^{t} y(\theta) z(\theta) d \theta,
\end{aligned}
$$


where $x_{0}=s / d$. Calculating the derivative of $W_{1}$ along positive solutions of system (3), it follows that

$$
\begin{aligned}
\frac{d W_{1}}{d t}= & \frac{\left(x-x_{0}\right)}{x}(s-d x-\beta x y+\delta w) \\
& +\frac{\delta}{(d+\eta+q) x_{0}}\left(x-x_{0}+w\right) \\
& \times[s-d x-(\eta+q) w] \\
& +\frac{(\delta+\eta+q)}{q}(q w-\alpha y-p y z)+\beta x y \\
& -(\delta+\eta+q) w+\frac{p(\delta+\eta+q)}{q} y z \\
& -\frac{p r(\delta+\eta+q)}{c q} z .
\end{aligned}
$$

On substituting $s=d x_{0}$ and $\delta w\left(x-x_{0}\right) / x=$ $-\delta w\left(\left(x-x_{0}\right)^{2} / x x_{0}\right)+\delta w\left(\left(x-x_{0}\right) / x_{0}\right)$ into (26), we derive that

$$
\begin{aligned}
\frac{d W_{1}}{d t}= & -\left(d x_{0}+\delta w+\frac{d \delta x}{d+\eta+q}\right) \\
& \times \frac{\left(x-x_{0}\right)^{2}}{x x_{0}}-\frac{\delta w^{2}(\eta+q)}{(d+\eta+q) x_{0}} \\
& +\frac{\alpha(\delta+\eta+q)}{q}\left(R_{0}-1\right) y-\frac{\operatorname{pr}(\delta+\eta+q)}{c q} z .
\end{aligned}
$$

If $R_{0}<1$, we have $d W_{1} / d t \leq 0$ for all $x>0, w>0, y>0$, and $z>0$. It follows that the uninfected equilibrium $E_{0}$ is stable. Clearly, it follows from (27) that $d W_{1} / d t=0$ if and only if $x=s / d, w=0, y=0$, and $z=0$. Therefore the largest invariant set in the set $\left\{(x, w, y, z) \in R_{+}^{4} \mid d W_{1} / d t=0\right\}$ is the singleton $\left\{E_{0}\right\}$. By LaSalle invariance principle [21], we can conclude that the equilibrium $E_{0}$ is globally asymptotically stable.

From the above analysis, we can obtain that the time delay has no effect on the stability of the uninfected equilibrium $E_{0}$ for the DDE model.

Theorem 8. For system (3), if $R_{1}<1$ and $1<R_{0} \leq 1+$ $(\eta+q) / \delta$, then $C T L-a b s e n t$ infection equilibrium $E_{1}$ is globally asymptotically stable.

Proof. Define a lyapunov functional

$$
\begin{aligned}
W_{2}(x, w, y, z)= & x-x_{1}-x_{1} \ln \frac{x}{x_{1}} \\
& +\frac{\beta x_{1} y_{1}}{q w_{1}}\left(y-y_{1}-y_{1} \ln \frac{y}{y_{1}}\right)
\end{aligned}
$$

$$
\begin{aligned}
& +\frac{\delta}{2(d+\eta+q) x_{1}}\left[\left(x-x_{1}\right)+\left(w-w_{1}\right)\right]^{2} \\
& +w-w_{1}-w_{1} \ln \frac{w}{w_{1}} \\
& +\frac{\beta p x_{1} y_{1}}{c q w_{1}} z+\frac{\beta p x_{1} y_{1}}{q w_{1}} \int_{t-\tau}^{t} y(\theta) z(\theta) d \theta .
\end{aligned}
$$

Calculating the derivative of $W_{2}$ along positive solutions of system (3), it follows that

$$
\begin{aligned}
\frac{d W_{2}}{d t}= & \left(1-\frac{x_{1}}{x}\right)(s-d x-\beta x y+\delta w) \\
& +\frac{\beta x_{1} y_{1}}{q w_{1}}\left(1-\frac{y_{1}}{y}\right)(q w-\alpha y-p y z) \\
& +\frac{\delta}{(d+\eta+q) x_{1}}\left[\left(x-x_{1}\right)+\left(w-w_{1}\right)\right] \\
& \times[s-d x-(\eta+q) w] \\
& +\left(1-\frac{w_{1}}{w}\right)[\beta x y-(\delta+\eta+q) w] \\
& -\frac{\beta p r x_{1} y_{1}}{c q w_{1}} z+\frac{\beta p x_{1} y_{1}}{q w_{1}} y z .
\end{aligned}
$$

At CTL-absent infection equilibrium $E_{1}$, on substituting $s=$ $\beta x_{1} y_{1}+d x_{1}-\delta w_{1}, \alpha=q\left(w_{1} / y_{1}\right)$, and $\delta+\eta+q=\beta x_{1} y_{1} / w_{1}$ into (29), we obtain that

$$
\begin{aligned}
& \frac{d W_{2}}{d t} \\
& =\left(1-\frac{x_{1}}{x}\right)\left[-d\left(x-x_{1}\right)-\beta x y+\beta x_{1} y_{1}+\delta\left(w-w_{1}\right)\right] \\
& +\frac{\beta x_{1} y_{1}}{q w_{1}}\left(1-\frac{y_{1}}{y}\right)\left(q w-q w_{1} \frac{y}{y_{1}}-p y z\right) \\
& +\frac{\delta}{(d+\eta+q)}\left[\left(x-x_{1}\right)+\left(w-w_{1}\right)\right] \\
& \quad \times\left[-d\left(x-x_{1}\right)-(\eta+q)\left(w-w_{1}\right)\right] \\
& +\left(1-\frac{w_{1}}{w}\right)\left(\beta x y-\beta x_{1} y_{1} \frac{w}{w_{1}}\right) \\
& -\frac{\beta r p x_{1} y_{1}}{c q w_{1}} z+\frac{\beta p x_{1} y_{1}}{q w_{1}} y z .
\end{aligned}
$$


It follows from (16) and (30) that

$$
\begin{aligned}
& \frac{d W_{2}}{d t}=-\left(d x_{1}+\delta\left(w-w_{1}\right)\right) \frac{\left(x-x_{1}\right)^{2}}{x x_{1}} \\
& +\frac{\delta}{x_{1}}\left(x-x_{1}\right)\left(w-w_{1}\right) \\
& +\beta x_{1} y_{1}\left(1-\frac{x y}{x_{1} y_{1}}-\frac{x_{1}}{x}+\frac{y}{y_{1}}\right) \\
& +\beta x_{1} y_{1}\left(\frac{w}{w_{1}}-\frac{y}{y_{1}}-\frac{y_{1} w}{y w_{1}}+1\right) \\
& -\frac{\beta x_{1} y_{1}}{q w_{1}}\left(1-\frac{y_{1}}{y}\right) p y z \\
& +\beta x_{1} y_{1}\left(\frac{x y}{x_{1} y_{1}}-\frac{w}{w_{1}}-\frac{x w_{1} y}{x_{1} w y_{1}}+1\right) \\
& -\frac{\delta}{x_{1}}\left[\frac{d}{d+\eta+q}\left(x-x_{1}\right)^{2}+\left(x-x_{1}\right)\right. \\
& \left.\times\left(w-w_{1}\right)+\frac{\eta+q}{d+\eta+q}\left(w-w_{1}\right)^{2}\right] \\
& -\frac{\beta r p x_{1} y_{1}}{c q w_{1}} z+\frac{\beta p x_{1} y_{1}}{q w_{1}} y z \\
& =-\left(d x_{1}-\delta w_{1}+\delta w+\frac{d \delta x}{d+\eta+q}\right) \\
& \times \frac{\left(x-x_{1}\right)^{2}}{x x_{1}}-\frac{\delta(\eta+q)}{(d+\eta+q)}\left(w-w_{1}\right)^{2} \\
& -\beta x_{1} y_{1}\left(\frac{x_{1}}{x}+\frac{y_{1} w}{y w_{1}}+\frac{x y w_{1}}{x_{1} y_{1} w}-3\right) \\
& +\frac{\beta p x_{1} y_{1}}{c q w_{1}}\left[\frac{d(\delta+\eta+q)+r \beta(\eta+q)}{\beta(\eta+q)}\right]\left(R_{1}-1\right) z \text {. }
\end{aligned}
$$

Since $x_{1} / x+y_{1} w / y w_{1}+x y w_{1} / x_{1} y_{1} w-3 \geq 0$ and the equality holds if and only if $x=x_{1}, w=w_{1}$, and $y=y_{1}$. If $R_{0} \leq$ $1+(\eta+q) / \delta$, then $d x_{1}-\delta w_{1} \geq 0$. Therefore, if $R_{1}<1$ and $1<$ $R_{0} \leq 1+(\eta+q) / \delta$, it follows from (31) that $d W_{2} / d t \leq 0$ for all $x>0, w>0, y>0$, and $z>0$. It is readily seen from (31) that $d W_{2} / d t=0$ if and only if $x=x_{1}, w=w_{1}, y=y_{1}$, and $z=0$. Thus the largest invariant set in the set $\left\{(x, w, y, z) \in R_{+}^{4}\right.$ । $\left.d W_{2} / d t=0\right\}$ is the singleton $\left\{E_{1}\right\}$. Then the global asymptotic stability of $E_{1}$ follows from the LaSalle's invariance principle [21].

From the above analysis, we obtain that the time delay has no effect on the stability of the CTL-absent infection equilibrium $E_{1}$ for the DDE model. Next, we analyze stability and Hopf bifurcation at the CTL-present equilibrium $E_{2}$.
Firstly, the linearized equations of system (3) at $E_{2}$ are given as follows:

$$
\begin{gathered}
\frac{d x}{d t}=-\left(\beta y_{2}+d\right) x(t)-\beta x_{2} y(t)+\delta w(t), \\
\frac{d w}{d t}=\beta y_{2} x(t)+\beta x_{2} y(t)-(\delta+\eta+q) w(t), \\
\frac{d y}{d t}=q w(t)-\left(\alpha+p z_{2}\right) y(t)-p y_{2} z(t), \\
\frac{d z}{d t}=c z_{2} y(t-\tau)+c y_{2} z(t-\tau)-r z(t) .
\end{gathered}
$$

The characteristic equation of system (32) at $O(0,0,0,0)$ takes the form

$$
\begin{aligned}
G(\lambda)= & \lambda^{4}+M_{1} \lambda^{3}+M_{2} \lambda^{2}+M_{3} \lambda+M_{4} \\
& -\left(N_{1} \lambda^{3}+N_{2} \lambda^{2}+N_{3} \lambda+N_{4}\right) e^{-\lambda \tau}=0,
\end{aligned}
$$

where

$$
\begin{aligned}
& M_{1}= \beta y_{2}+d+\delta+\eta+q+\alpha+p z_{2}+r, \\
& M_{2}= \beta y_{2}(\eta+q)+d(\delta+\eta+q) \\
&+\left(\beta y_{2}+d\right)\left(\alpha+p z_{2}\right) \\
&+r\left(\beta y_{2}+d+\delta+\eta+q+\alpha+p z_{2}\right), \\
& M_{3}= r\left[\beta y_{2}(\eta+q)+d(\delta+\eta+q)\right. \\
&\left.+\left(\beta y_{2}+d\right)\left(\alpha+p z_{2}\right)\right] \\
&+\beta y_{2}(\eta+q)\left(\alpha+p z_{2}\right), \\
& M_{4}= r \beta y_{2}(\eta+q)\left(\alpha+p z_{2}\right), \\
& \quad N_{1}=r \\
& N_{2}= r\left(\beta y_{2}+d+\delta+\eta+q+\alpha+p z_{2}\right) \\
&-c p y_{2} z_{2}, \\
& N_{3}= r\left[\beta y_{2}(\eta+q)+d(\delta+\eta+q)\right. \\
&\left.+\left(\beta y_{2}+d\right)\left(\alpha+p z_{2}\right)\right] \\
&-c p y_{2} z_{2}\left(\beta y_{2}+d+\delta+\eta+q\right), \\
& N_{4}=r \beta y_{2}(\eta+q)\left(\alpha+p z_{2}\right) \\
&-c p y_{2} z_{2}\left[\beta y_{2}(\eta+q)+d(\delta+\eta+q)\right] .
\end{aligned}
$$

Theorem 9. Suppose $\tau=0$, if $R_{1}>1$, then the CTL-present equilibrium $E_{2}$ of system (3) is locally asymptotically stable.

Proof. If $\tau=0$, (33) becomes

$$
\begin{aligned}
\lambda^{4}+ & \left(M_{1}-N_{1}\right) \lambda^{3}+\left(M_{2}-N_{2}\right) \lambda^{2} \\
& +\left(M_{3}-N_{3}\right) \lambda+M_{4}-N_{4}=0 .
\end{aligned}
$$


Since $R_{1}>1, x_{2}>0, w_{2}>0, y_{2}>0$, and $z_{2}>0$, by the Routh-Hurwitz criteria, it follows that

$$
\begin{aligned}
& H_{1}=M_{1}-N_{1}=\beta y_{2}+d+\delta+\eta+q+\alpha+p z_{2}>0 \text {, } \\
& H_{2}=\left(M_{1}-N_{1}\right)\left(M_{2}-N_{2}\right)-\left(M_{3}-N_{3}\right) \\
& =\left[\beta y_{2}(\eta+q)+d(\delta+\eta+q)\right. \\
& \left.+\left(\beta y_{2}+d\right)\left(\alpha+p z_{2}\right)+c p y_{2} z_{2}\right] \\
& \times\left(\beta y_{2} d+\delta+\eta+q+\alpha+p z_{2}\right) \\
& -\beta y_{2}(\eta+q)\left(\alpha+p z_{2}\right) \\
& -c p y_{2} z_{2}\left(\beta y_{2}+d+\delta+\eta+q\right) \\
& =\left[d(\delta+\eta+q)+\left(\beta y_{2}+d\right)\left(\alpha+p z_{2}\right)\right] \\
& \times\left[\beta y_{2}+d+\delta+\eta+q+\alpha+p z_{2}\right] \\
& +\beta y_{2}(\eta+q)\left(\beta y_{2}+d+\delta+\eta+q\right) \\
& +c p y_{2} z_{2}\left(\alpha+p z_{2}\right)>0 \text {, } \\
& H_{3}=\left(M_{1}-N_{1}\right)\left[\left(M_{2}-N_{2}\right)\left(M_{3}-N_{3}\right)\right. \\
& \left.-\left(M_{1}-N_{1}\right)\left(M_{4}-N_{4}\right)\right]-\left(M_{3}-N_{3}\right)^{2} \\
& =\left[\beta y_{2}(\eta+q)+d(\delta+\eta+q)\right. \\
& \left.+\left(\beta y_{2}+d\right)\left(\alpha+p z_{2}\right)+c p y_{2} z_{2}\right] \\
& \times\left(\beta y_{2}+d+\delta+\eta+q+\alpha+p z_{2}\right) \\
& \times\left[\beta y_{2}(\eta+q)\left(\alpha+p z_{2}\right)\right. \\
& \left.+c p y_{2} z_{2}\left(\beta y_{2}+d+\delta+\eta+q\right)\right] \\
& -\left(\beta y_{2}+d+\delta+\eta+q+\alpha+p z_{2}\right)^{2} \\
& \times c p y_{2} z_{2}\left[\beta y_{2}(\eta+q)+d(\delta+\eta+q)\right] \\
& -\left[\beta y_{2}(\eta+q)\left(\alpha+p z_{2}\right)\right. \\
& \left.+c p y_{2} z_{2}\left(\beta y_{2}+d+\delta+\eta+q\right)\right]^{2} \\
& =A\left(c p y_{2} z_{2}\right)^{2}+B c p y_{2} z_{2}+C \text {, }
\end{aligned}
$$

where

$$
\begin{aligned}
A= & \left(\alpha+p z_{2}\right)\left(\beta y_{2}+d+\delta+\eta+q\right)>0, \\
B= & {\left[\left(\beta y_{2}+d\right)^{2}+\beta y_{2} \delta\right]\left(\alpha+p z_{2}\right) } \\
& \times\left(\beta y_{2}+d+\alpha+p z_{2}\right)+\left[\left(\beta y_{2}+d\right)^{2}+\beta y_{2} \delta\right] \\
& \times \delta\left(\alpha+p z_{2}\right)+\left(\beta d y_{2}+d^{2}\right)(\eta+q)\left(\alpha+p z_{2}\right) \\
& -\beta y_{2}(\eta+q)^{2}\left(\alpha+p z_{2}\right),
\end{aligned}
$$

$$
\begin{aligned}
C= & \beta y_{2}(\eta+q)\left(\alpha+p z_{2}\right)\left(\beta y_{2}+d+\delta+\eta+q\right) \\
& \times\left[\beta y_{2}(\eta+q)+d(\delta+\eta+q)\left(\beta y_{2}+d\right)\left(\alpha+p z_{2}\right)\right] \\
& +\beta y_{2}(\eta+q)\left(\alpha+p z_{2}\right)^{2} \\
& \times\left[d(\delta+\eta+q)+\left(\beta y_{2}+d\right)\left(\alpha+p z_{2}\right)\right]>0 .
\end{aligned}
$$

Let

$$
\begin{gathered}
D=\beta y_{2}(\eta+q)^{2}\left(\alpha+p z_{2}\right), \quad h=c p y_{2} z_{2}>0, \\
f(h)=A h^{2}+B h+C \\
g(h)=A h^{2}-D h+C .
\end{gathered}
$$

If $g(h)>0$ for all $h>0$, then $f(h)>0$. Since

$$
\begin{aligned}
\Delta_{1}= & D^{2}-4 A C \\
= & -4\left(\alpha+p z_{2}\right)^{3}\left(\beta y_{2}+d+\delta+\eta+q\right) \beta y_{2}(\eta+q) \\
& \times\left[d(\delta+\eta+q)+\left(\beta y_{2}+d\right)\left(\alpha+p z_{2}\right)\right] \\
& -4\left(\alpha+p z_{2}\right)^{2}\left(\beta y_{2}+d+\delta\right) \beta y_{2}(\eta+q) \\
& \times\left(\beta y_{2}+d+\delta+\eta+q\right) \\
& \times\left[\beta y_{2}(\eta+q)\right. \\
& \left.+d(\delta+\eta+q)\left(\beta y_{2}+d\right)\left(\alpha+p z_{2}\right)\right] \\
& -4\left(\alpha+p z_{2}\right)^{3}(\eta+q)^{2} \beta y_{2}\left(\beta y_{2}+d+\delta+\eta+q\right) \\
& \times d(\delta+\eta+q)\left(\beta y_{2}+d\right) \\
& -4\left(\alpha+p z_{2}\right)^{2}(\eta+q)^{3}\left(\beta y_{2}\right)^{2}\left(\beta y_{2}+d+\delta\right) \\
& -3\left[\beta y_{2}(\eta+q)^{2}\left(\alpha+p z_{2}\right)\right]^{2}<0,
\end{aligned}
$$

and $g(0)=C>0$, it follows that $g(h)>0$ for all $h>0$, thus $f(h)>0$, that is, $H_{3}>0$.

$$
\begin{aligned}
H_{4} & =\left|\begin{array}{cccc}
M_{1}-N_{1} & M_{3}-N_{3} & 0 & 0 \\
1 & M_{2}-N_{2} & M_{4}-N_{4} & 0 \\
0 & M_{1}-N_{1} & M_{3}-N_{3} & 0 \\
0 & 1 & M_{2}-N_{2} & M_{4}-N_{4}
\end{array}\right| \\
& =\left(M_{4}-N_{4}\right) H_{3} .
\end{aligned}
$$

Noting that

$$
M_{4}-N_{4}=c p y_{2} z_{2}\left[\beta y_{2}(\eta+q)+d(\delta+\eta+q)\right]>0,
$$

it follows that $H_{4}>0$. Therefore, all the roots of (35) have negative real parts. This completes the proof of Theorem 9.

For $\tau=0$, the all roots of $G(\lambda)=0$ have negative real roots in Theorem 9. By the continuous dependence of roots 
of $G(\lambda)=0$ on the parameters, it follows that there exists $\bar{\tau}>0$ such that for $\tau \in[0, \bar{\tau})$, all roots of (33) satisfy

$$
G(\lambda)=0, \quad \operatorname{Re}(\lambda)<0, \quad \text { for } \tau \in[0, \bar{\tau}),
$$

and when $\tau=\bar{\tau}, \operatorname{Re}(\lambda)=0$. To determine $\bar{\tau}$ and the associated purely imaginary roots $\bar{\omega} i(\bar{\omega}>0)$.

Suppose that $\lambda=\omega i(\omega>0)$ is a solution of (33), it follows that

$$
\begin{aligned}
\omega^{4} & -M_{1} \omega^{3} i-M_{2} \omega^{2} i+M_{3} \omega i \\
& -\left(-N_{1} \omega^{3} i-N_{2} \omega^{2}+B_{3} \omega i+B_{4}\right)(\cos \omega \tau-i \sin \omega \tau)=0 .
\end{aligned}
$$

Separating the real and imaginary parts, it follows that

$$
\begin{aligned}
& \left(N_{4}-N_{2} \omega^{2}\right) \cos \omega \tau+\left(N_{3} \omega-N_{1} \omega^{3}\right) \sin \omega \tau \\
& \quad=\omega^{4}-M_{2} \omega^{2}+M_{4}, \\
& \left(N_{1} \omega^{3}-N_{3} \omega\right) \cos \omega \tau+\left(N_{4}-N_{2} \omega^{2}\right) \sin \omega \tau \\
& \quad=M_{1} \omega^{3}-M_{3} \omega .
\end{aligned}
$$

From (44), we obtain that

$$
\begin{aligned}
& \cos \omega \tau=\frac{1}{\Delta}\left(A_{1} \omega^{6}+A_{2} \omega^{4}+A_{3} \omega^{2}+A_{4}\right), \\
& \sin \omega \tau=-\frac{\omega}{\Delta}\left(B_{1} \omega^{6}+B_{2} \omega^{4}+B_{3} \omega^{2}+B_{4}\right),
\end{aligned}
$$

where

$$
\begin{gathered}
A_{1}=M_{1} N_{1}-N_{2}, \\
A_{2}=N_{4}+M_{2} N_{2}-M_{1} N_{3}-M_{3} N_{1}, \\
A_{3}=M_{3} N_{3}-M_{2} N_{4}-M_{4} N_{2}, \quad A_{4}=M_{4} N_{4}, \\
B_{1}=N_{1}, \quad B_{2}=M_{1} N_{2}-N_{3}-M_{2} N_{1}, \\
B_{3}=M_{2} N_{3}+M_{4} N_{1}-M_{3} N_{2}-M_{1} N_{4}, \\
B_{4}=M_{3} N_{4}-M_{4} N_{3}, \\
\Delta=\left(N_{4}-N_{2} \omega^{2}\right)^{2}+\left(N_{3} \omega-N_{1} \omega^{3}\right)^{2}>0 .
\end{gathered}
$$

It follows from (44) that

$$
\omega^{8}+q_{1} \omega^{6}+q_{2} \omega^{4}+q_{3} \omega^{2}+q_{4}=0,
$$

where

$$
\begin{gathered}
q_{1}=M_{1}^{2}-2 M_{2}-N_{1}^{2}, \\
q_{2}=M_{2}^{2}+2 M_{4}-2 M_{1} M_{3}+2 N_{1} N_{3}-M_{2}^{2}, \\
q_{3}=M_{3}^{2}-2 M_{2} M_{4}+2 N_{2} N_{4}-M_{3}^{2}, \quad q_{4}=M_{4}^{2}-N_{4}^{2} .
\end{gathered}
$$

Letting $u=\omega^{2}$, (47) becomes

$$
u^{4}+q_{1} u^{3}+q_{2} u^{2}+q_{3} u+q_{4}=0 .
$$

Denote $G(u)=u^{4}+q_{1} u^{3}+q_{2} u^{2}+q_{3} u+q_{4}$. Then we have

$$
G^{\prime}(u)=4 u^{3}+3 q_{1} u^{2}+2 q_{2} u+q_{3} .
$$

Suppose that (49) has positive real roots. Without loss of generality, we assume that it has $n(1 \leq n \leq 4)$ positive real roots, defined by $u_{1}<u_{2}<\cdots<u_{n}$, respectively. Then (47) has $n$ positive real roots

$$
\omega_{1}=\sqrt{u_{1}}, \omega_{2}=\sqrt{u_{2}}, \ldots, \omega_{n}=\sqrt{u_{n}} .
$$

From (45), we have

$$
\tau_{l}^{j}=\frac{1}{\omega_{l}}\left(\arccos \frac{A_{1} \omega_{l}^{6}+A_{2} \omega_{l}^{4}+A_{3} \omega_{l}^{2}+A_{4}}{\left(N_{4}-N_{2} \omega_{l}^{2}\right)^{2}+\left(N_{3} \omega_{l}-N_{1} \omega_{l}^{3}\right)^{2}}+2 j \pi\right),
$$

where $l=1,2, \ldots, n, j=0,1,2,3, \ldots$, then $\pm \omega_{l}$ i are a pair of purely imaginary roots of (33) with $\tau_{1}^{j}$.

Differentiating (33) implicitly with respect to $\tau$, we obtain that

$$
\begin{aligned}
{\left[\frac{d \lambda}{d \tau}\right]^{-1}=} & \frac{-\left(4 \lambda^{3}+3 M_{1} \lambda^{2}+2 M_{2} \lambda+M_{3}\right) e^{\lambda \tau}}{\lambda\left(N_{1} \lambda^{3}+N_{2} \lambda^{2}+N_{3} \lambda+N_{4}\right)} \\
& +\frac{3 N_{1} \lambda^{2}+2 N_{2} \lambda+N_{3}}{\lambda\left(N_{1} \lambda^{3}+N_{2} \lambda^{2}+N_{3} \lambda+N_{4}\right)}-\frac{\tau}{\lambda} .
\end{aligned}
$$

Thus,

$$
\begin{aligned}
\operatorname{Re}\left[\frac{d \lambda}{d \tau}\right]_{\tau=\tau_{l}^{j}}^{-1}=\frac{1}{\omega_{l} \Delta}\{ & \left(3 M_{1} \omega_{l}^{2}-M_{3}\right) \\
\times & {\left[\left(N_{1} \omega_{l}^{3}-N_{3} \omega_{l}\right) \cos \omega_{l} \tau\right.} \\
& \left.+\left(N_{4}-N_{4} \omega_{l}^{2}\right) \sin \omega_{l} \tau\right] \\
+ & \left(4 \omega_{l}^{3}-2 M_{2} \omega_{l}\right) \\
\times & {\left[\left(N_{4}-N_{2} \omega_{l}^{2}\right) \cos \omega_{l} \tau\right.} \\
& \left.-\left(N_{1} \omega_{l}^{3}-N_{3} \omega_{l}\right) \sin \omega_{l} \tau\right] \\
+ & \left(N_{3}-3 N_{1} \omega_{l}^{2}\right)\left(N_{1} \omega_{l}^{3}-N_{3} \omega_{l}\right) \\
+ & \left.2 N_{2} \omega_{l}\left(N_{4}-N_{2} \omega_{l}^{2}\right)\right\} .
\end{aligned}
$$

On substituting (45) into (54), we obtain

$$
\begin{aligned}
\operatorname{Re}\left[\frac{d \lambda}{d \tau}\right]_{\tau=\tau_{l}^{j}}^{-1} & \\
=\frac{1}{\Delta}[ & 4 \omega_{l}^{6}+3\left(M_{1}^{2}-2 M_{2}-N_{1}^{2}\right) \omega_{l}^{4} \\
& +2\left(M_{2}^{2}-N_{2}^{2}+2 M_{4}+2 N_{1} N_{3}-2 M_{1} M_{3}\right) \omega_{l}^{2} \\
& \left.+M_{3}^{2}-N_{3}^{2}+2 N_{2} N_{4}-2 M_{2} M_{4}\right]=\frac{G^{\prime}\left(u_{l}\right)}{\Delta},
\end{aligned}
$$


where $\Delta=\left(N_{4}-N_{2} \omega^{2}\right)^{2}+\left(N_{3} \omega-N_{1} \omega^{3}\right)^{2}>0$. If we suppose that $G^{\prime}(u) \neq 0$, then

$$
\begin{aligned}
\operatorname{sign}\left\{\operatorname{Re}\left[\frac{d \lambda}{d \tau}\right]_{\tau=\tau_{l}^{j}}\right\} & =\operatorname{sign}\left\{\operatorname{Re}\left[\frac{d \lambda}{d \tau}\right]_{\tau=\tau_{l}^{j}}^{-1}\right\} \\
& =\operatorname{sign}\left\{\frac{G^{\prime}\left(u_{l}\right)}{\Delta}\right\}=\operatorname{sign}\left\{G^{\prime}\left(u_{l}\right)\right\} .
\end{aligned}
$$

Applying Theorem 9 and the Hopf bifurcation theorem for functional differential equation [22] from (56), we derive the existence of a Hopf bifurcation as follows.

Theorem 10. Suppose that (49) has at least one simple positive root and $\bar{u}$ is the last such root, then there is a Hopf bifurcation for the system (3) as $\tau$ passes upwards through $\bar{\tau}$ leading to a periodic solution that bifurcates from $E_{2}$, where

$$
\bar{\tau}=\frac{1}{\bar{\omega}}\left(\arccos \frac{A_{1} \bar{\omega}_{l}^{6}+A_{2} \bar{\omega}_{l}^{4}+A_{3} \bar{\omega}_{l}^{2}+A_{4}}{\left(N_{4}-N_{2} \bar{\omega}_{l}^{2}\right)^{2}+\left(N_{3} \bar{\omega}_{l}-N_{1} \bar{\omega}_{l}^{3}\right)^{2}}+2 j \pi\right) .
$$

Remark 11. If $\bar{u}$ is the last simple positive root of (49), then we have $G^{\prime}(\bar{u})>0$. From (56), we obtain $\operatorname{Re}[d \lambda / d \tau]_{\tau=\bar{\tau}}>0$.

Remark 12. In this paper, we construct a few Lyapunov functions (functionals) to prove the global stability of steady states of ODE model (DDE model). This function (functional) can also prove the global stability of steady states of other viral infections models with cure rate $[7,10]$. Moreover, the method studying the existence of Hopf bifurcations applies to other viral infections models with immune delay $[2,18,19]$.

\section{Numerical Simulations}

In this section, we perform numerical calculation to support our theoretical analysis of this paper.

Example 13. If we choose parameters $s=1.5, \beta=0.3, \alpha=$ $0.1, p=0.015, c=0.12, r=0.8, d=0.1, \delta=0.6, \eta=0.4$, and $q=0.7$, then $R_{1}=1.33>1$ and the CTL-present equilibrium $E_{2}(1.1751,1.3825,6.6667,1.6283)$. From (49), we obtain that

$$
u^{4}+10.5385 u^{3}+3.9615 u^{2}+0.0729 u+0.0151=0 .
$$

Equation (58) has no positive roots, and all roots have negative real parts. Therefore, the equilibrium is locally asymptotically stable for all $\tau \geq 0$ (e.g., $\tau=1.5$, see Figure 1 ).

Example 14. If we select parameters $s=10, \beta=0.1, \alpha=$ $0.4, p=0.3, c=0.5, r=0.9, d=1, \delta=1, \eta=1$, and $q=4$, then $R_{1}=1.45>1$ and the CTL-present equilibrium $E_{2}(8.8107,0.2642,1.7997,0.6244)$. It follows from (49) that

$$
u^{4}+40.7439 u^{3}+41.9038 u^{2}-1.9589 u-2.0726=0 .
$$

Equation (59) has only one positive real root $u=$ 0.2217 and any other roots have negative real parts. Thus,



FIGURE 1: The CTL-present infection equilibrium $E_{2}$ of system (3) is locally asymptotically stable when $R_{1}=1.33>1$ and $\tau=1.5$.

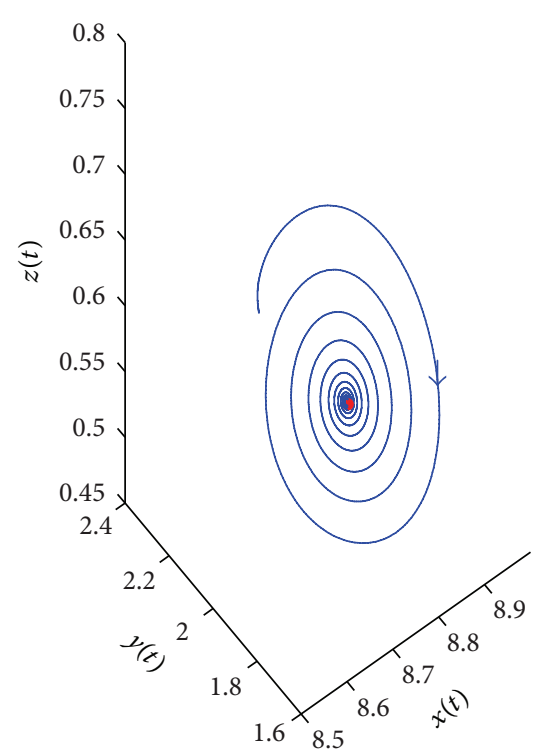

FIGURE 2: The CTL-present infection equilibrium $E_{2}$ of system (3) is locally asymptotically stable when $R_{1}=1.45>1$ and $\tau=0.2<\bar{\tau}$.

$\bar{\omega}=\sqrt{u}=0.4709$. In addition, it is easy to show that $\bar{\tau}=0.948$. Therefore, Theorem 10 is satisfied.

If $\tau=0.2<\bar{\tau}$, the CTL-present infection equilibrium $E_{2}$ of system (3) is locally asymptotically stable (see Figure 2 ). If $\tau=5>\bar{\tau}$, then the CTL-present infection equilibrium $E_{2}$ of system (3) becomes unstable, and the Hopf bifurcation occurs (see Figure 3).

\section{Conclusion}

In this paper, we have studied an HIV infection model including infected cells in an eclipse stage and CTL immune 


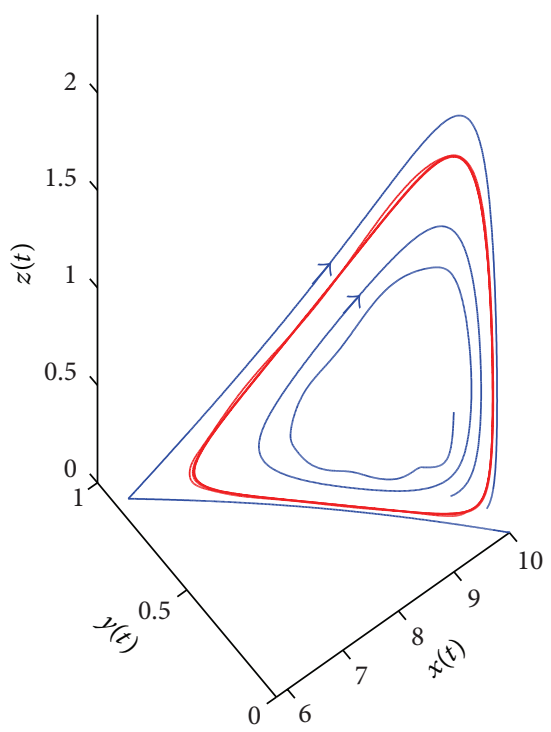

FIGURE 3: The bifurcating periodic solution from the equilibrium $E_{2}$ of system (3) occurs when $\tau=5$.

response. The global stability of the uninfected equilibrium $E_{0}$ for system (2) and (3) has been given by the LaSalle's invariance principle when the basic reproductive ratio $R_{0}<1$; it shows that the disease will be controlled. Compared with the earlier modeling studies on the immune response of HIV infection $[14,18,20]$, our analysis reveals the existence of a CTL-absent infection equilibrium $E_{1}\left(x_{1}, w_{1}, y_{1}, 0\right)$ when $R_{0}>1$. We also obtained the global asymptotic stability of a CTL-absent infection equilibrium $E_{1}$ for system (2) and (3) when $R_{1}<1$ and $1<R_{0}<1+(\eta+q) / \delta$. This indicates that there is a persistent HIV infection with no humeral and cellular immune responses. Furthermore, we can see that the time delay has no effect on the stability of the uninfected equilibrium $E_{0}$ and CTL-absent infection equilibrium $E_{1}$ for the DDE model.

When $R_{1}>1$, we show that the CTL-present infection equilibrium $E_{2}$ is locally asymptotically stable when the delay $\tau$ is small, and with the increase of the delay $\tau$ the stability of $E_{2}$ may destabilize and lead to Hopf bifurcation. This suggests that, with the HIV infection developing, the proviral load and CTL frequency can either stabilize at a constant level or show oscillations. Similar phenomenon was also observed in [17-19]. The HIV dynamics model without immune delay is globally stable $[14,15]$. In this paper, we show that the HIV infection model including infected cells in an eclipse stage and CTL immune response without immune delay is globally stable; and for the model with immune delay, Hopf bifurcation appears under some conditions.

\section{Acknowledgments}

This work was supported by National Natural Science Foundation of China $(61174209,11071013)$, the Basic Scientific Research Foundation of Central University (FRF-BR11-048B, FRF-BR-12-004), and the Basic Theory Research
Foundation for Engineering Research Institute of USTB (YJ2012-001).

\section{References}

[1] A. S. Perelson, P. Essunger, Y. Cao et al., "Decay characteristics of HIV-1-infected compartments during combination therapy," Nature, vol. 387, no. 6629, pp. 188-191, 1997.

[2] H. Zhu and X. Zou, "Impact of delays in cell infection and virus production on HIV-1 dynamics," Mathematical Medicine and Biology, vol. 25, no. 2, pp. 99-112, 2008.

[3] A. S. Perelson and P. W. Nelson, "Mathematical analysis of HIV1 dynamics in vivo," SIAM Review, vol. 41, no. 1, pp. 3-44, 1999.

[4] X. Zhou, X. Song, and X. Shi, "Analysis of stability and Hopf bifurcation for an HIV infection model with time delay," Applied Mathematics and Computation, vol. 199, no. 1, pp. 23-38, 2008.

[5] P. W. Nelson and A. S. Perelson, "Mathematical analysis of delay differential equation models of HIV-1 infection," Mathematical Biosciences, vol. 179, no. 1, pp. 73-94, 2002.

[6] E. Zintzaras and A. Kowald, "A mathematical model of HIV dynamics in the presence of a rescuing virus with replication deficiency," Theory in Biosciences, vol. 130, no. 2, pp. 127-134, 2011.

[7] C. Vargas-De-León, "Stability analysis of a model for HBV infection with cure of infected cells and intracellular delay," Applied Mathematics and Computation, vol. 219, no. 1, pp. 389398, 2012.

[8] L. Rong, M. A. Gilchrist, Z. Feng, and A. S. Perelson, "Modeling within-host HIV-1 dynamics and the evolution of drug resistance: trade-offs between viral enzyme function and drug susceptibility," Journal of Theoretical Biology, vol. 247, no. 4, pp. 804-818, 2007.

[9] A. S. Perelson, D. Kirschner, and R. De Boer, "Dynamics of HIV infection of CD $4^{+}$T cells," Mathematical Biosciences, vol. 114, no. 1, pp. 81-125, 1993.

[10] B. Buonomo and C. Vargas-De-León, "Global stability for an HIV-1 infection model including an eclipse stage of infected cells," Journal of Mathematical Analysis and Applications, vol. 385, no. 2, pp. 709-720, 2012.

[11] A. S. Peterson, A. P. Reid, S. Kim, and R. F. Siliciano, "Treatment implications of the latent reservoir for HIV-1," Advances in Pharmacology, vol. 55, pp. 411-425, 2007.

[12] Y. Ji, L. Min, Y. Zheng, and Y. Su, "A viral infection model with periodic immune response and nonlinear CTL response," Mathematics and Computers in Simulation, vol. 80, no. 12, pp. 2309-2316, 2010.

[13] M. A. Nowak and C. R. M. Bangham, "Population dynamics of immune responses to persistent viruses," Science, vol. 272, no. 5258, pp. 74-79, 1996.

[14] K. Wang, W. Wang, and X. Liu, "Global stability in a viral infection model with lytic and nonlytic immune responses," Computers \& Mathematics with Applications, vol. 51, no. 9-10, pp. 1593-1610, 2006.

[15] R. Culshaw, S. Ruan, and R. J. Spiteri, "Optimal HIV treatment by maximising immune response," Journal of Mathematical Biology, vol. 48, no. 5, pp. 545-562, 2004.

[16] K. Wang, W. Wang, H. Pang, and X. Liu, "Complex dynamic behavior in a viral model with delayed immune response," Physica D, vol. 226, no. 2, pp. 197-208, 2007.

[17] Q. Xie, D. Huang, S. Zhang, and J. Cao, "Analysis of a viral infection model with delayed immune response," Applied Mathematical Modelling, vol. 34, no. 9, pp. 2388-2395, 2010. 
[18] H. Zhu, Y. Luo, and M. Chen, "Stability and Hopf bifurcation of a HIV infection model with CTL-response delay," Computers \& Mathematics with Applications, vol. 62, no. 8, pp. 3091-3102, 2011.

[19] X. Song, S. Wang, and X. Zhou, "Stability and Hopf bifurcation for a viral infection model with delayed non-lytic immune response," Journal of Applied Mathematics and Computing, vol. 33, no. 1-2, pp. 251-265, 2010.

[20] D. Wodarz, J. P. Christensen, and A. R. Thomsen, "The importance of lytic and nonlytic immune response in viral infections," Trends in Immunology, vol. 23, no. 4, pp. 194-200, 2002.

[21] Y. Kuang, Delay Differential Equations with Applications in Population Dynamics, vol. 191 of Mathematics in Science and Engineering, Academic Press, San Diego, Calif, USA, 1993.

[22] J. Hale and S. M. V. Lunel, Introduction to Functional Differential Equations, Springer, New York, NY, USA, 1993. 


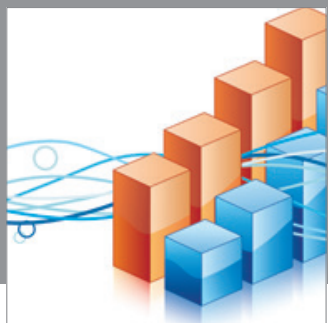

Advances in

Operations Research

mansans

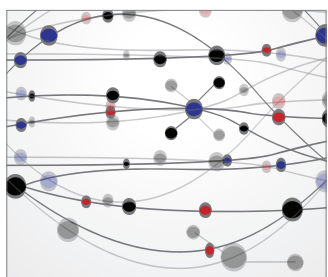

The Scientific World Journal
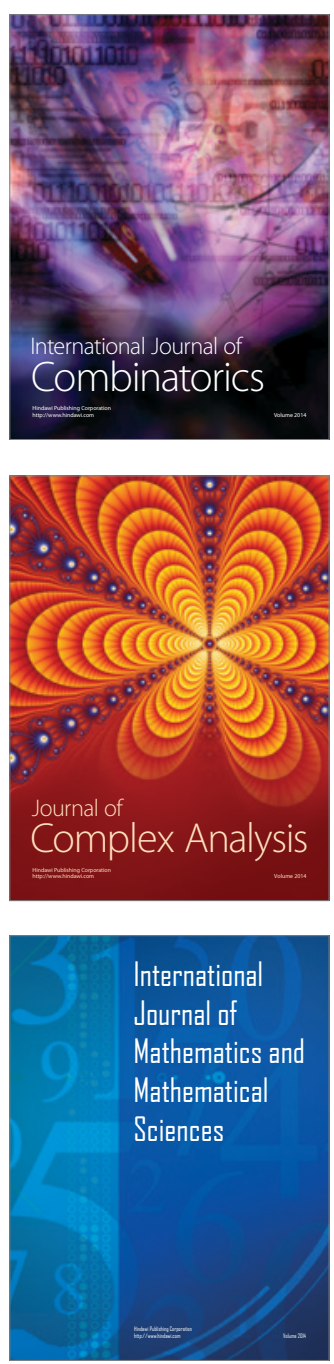
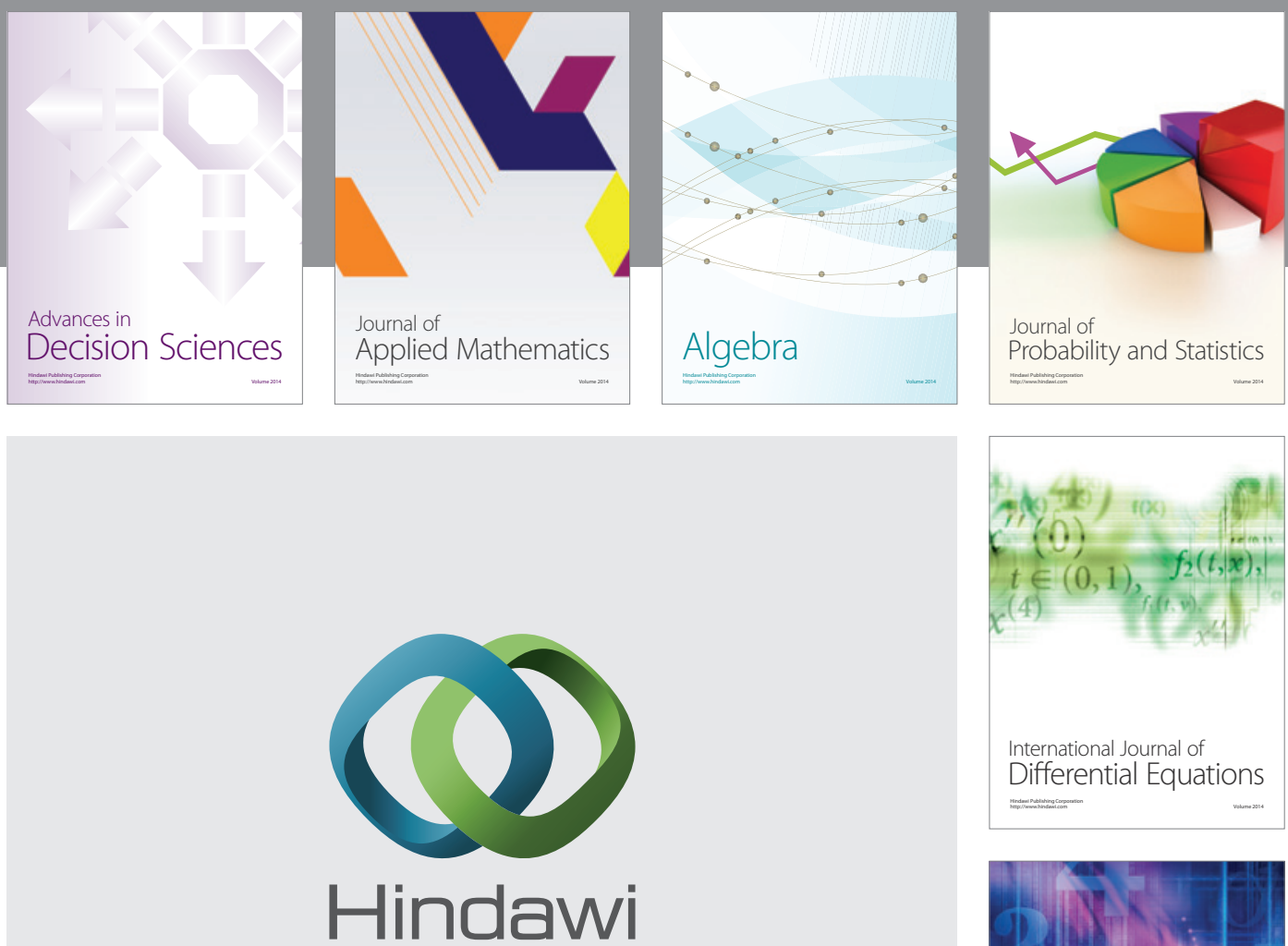

Submit your manuscripts at http://www.hindawi.com
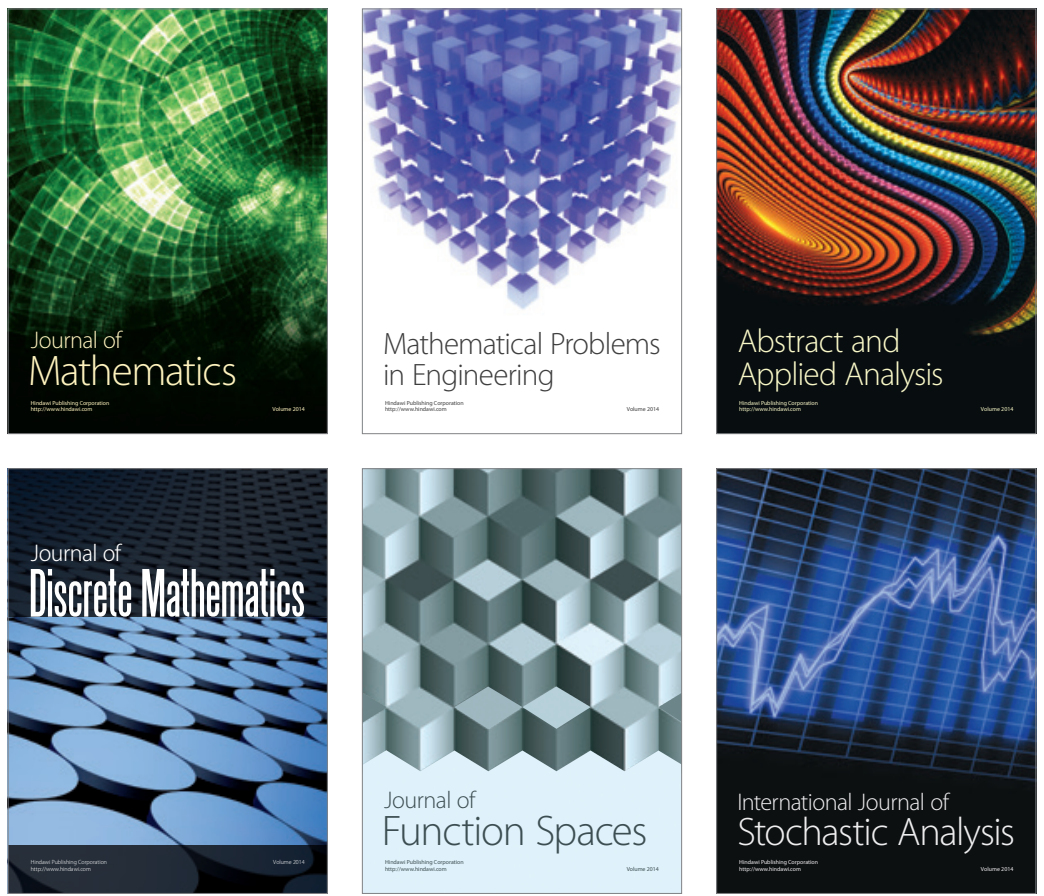

Journal of

Function Spaces



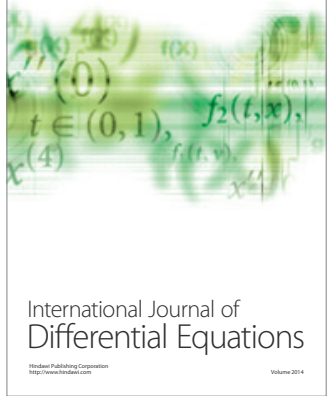
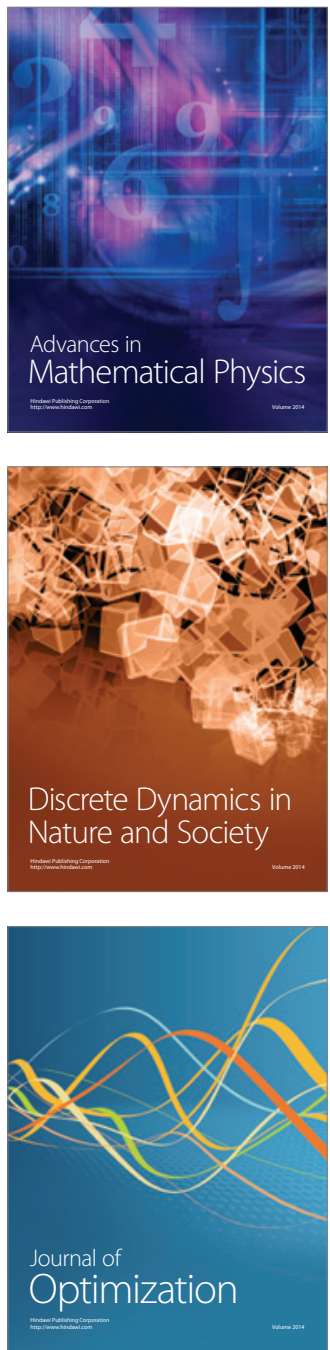